\section{Spin-Orbit Coupling and the Extraordinary Hall Effect}

IN order to find whether spin-orbit interaction gives an adequate explanation of the extraordinary Hall effect in ferromagnetics, in particular in its dependence on temperature, we have treated this problem quantum-mechanically. An elementary classical calculation using as Hamiltonian

$$
p^{2} / 2 m+V(r)+e \mathbf{E} \cdot \mathbf{r}+\left(1 / 2 m^{2} c^{2}\right) \mathbf{s} \cdot(\operatorname{grad} V \wedge \mathbf{p})
$$

shows that, to the first order in the spin orbit coupling constant, the motion of an electron with spin along the $z$-axis is the same as that in a fictitious magnetic field of components $(s / 2 \mathrm{emc})\left(-\partial^{2} V / \partial x \partial z,-\partial^{2} V / \partial y \partial z\right.$, $\partial V / \partial x^{2}+\partial^{2} V\left(\partial y^{2}\right)$. In a lattice where, on the average, the first two components are zero, and, with certain types of symmetry, in which $\partial^{2} V / \partial x^{2}+\partial^{2} V / \partial y^{2}$ might be replaced by $-(8 \pi / 3) p$ where $p$ is the mean charge density met by the electron, the equivalent 'magnetic field' would be of the order of magnitude $4 \pi s p / 3 e m c=4 \pi \rho M / 3 e^{2}$ where $M$ is the probable $z$-component of magnetic moment per electron. This is numerically quite sufficient for the purpose, but of course the magnitude depends very strongly on the path of the electron which governs the values of 0 which it meets.

Qualitatively the term proportional to s. (grad $V \wedge \mathbf{p})$ which occurs as part of the potential energy in the Schrödinger one-electron wave equation can be interpreted as follows. Suppose that the primary current is in the direction of the $x$-axis and that we consider spin eigenstates of the electron parallel and antiparallel to the $z$-axis. If we consider the variation of potential energy with $y$ in the neighbourhood of a row of lattice ions parallel to the $x$-axis along, say, the line $y=z=0$, and call regions $\mathrm{I}$ and II those regions near the $x y$ plane for which $y$ is respectively positive and negative and not greater than half the distance between neighbouring rows of ions, then, for electrons in the $\frac{1}{2}+\hbar$ eigenstate of $s z$, this term in the potential energy is negative in region $I$ and positive in region II. Provided that the square of the modulus of the electron wave function is greatest in the neighbourhood of the ions rather than in the region half way between them, the dynamic effect of this addition to the potential energy is very similar to that of the potential energy due to an electric field parallel to the $y$-axis. The transverse field serves to compensate for this effect and to annul current flow in the direction of the $y$-axis.

In a more detailed quantum mechanical treatment we have used the expansion of a one-electron wavefunction in terms of Bloch functions. The coefficients in this expension vary from their initial values in a manner determined partly by the applied electric field and the spin-orbit coupling. These coefficients can be used to evaluate the quantum-theoreticel current density and also to find the distribution function ${ }^{1}$, modified as it is by the presence of the electric field and the spin-orbit coupling.

The initial states of the electrons we have chosen as a superposition of $s$ - and $d$-states with random initial phases in which the $d$-states have a spin distribution in accordance with the magnetization. The resultant current distribution is obtained by supposing that the probability of a time $t$ elapsing between successive collisions with the lattice is proportional to $\exp (-t / \tau)$, the collision time, $\tau$, being, of course, dependent on temperature.
It is found that only interband matrix elements of the spin-orbit coupling contribute to the effect. The result contains as a factor the square of the resistivity. This is simply a consequence of the fact that the equations :

$$
J_{x}=\sigma_{x x} E_{x}+\sigma_{x y} E_{y}, \quad J_{y}=\sigma_{y x} E_{x}+\sigma_{y v} E_{y}
$$

connecting current $\mathbf{J}$ and electric field $\mathbf{E}$ give, when $J_{y}=0, E_{y} / J_{x}=-\sigma_{y x} /\left(\sigma_{x x x} \sigma_{y y}-\sigma_{x y} \sigma_{y x}\right)$. Since the second term in the denominator is much smaller than the first, and since we may for many lattices put $\sigma_{x x} \sigma_{y y}=\sigma^{2}$, we obtain the factor $\left(1 / \sigma^{2}\right)$, that is to say, the square of the resistivity. Karplus and Luttinger ${ }^{2}$ also obteined this factor unavoidably by considering $E_{y}=0, J_{y} \neq 0$, which, strictly speaking, is not the condition realized experimentally. However, in our result another factor occurs which is dependent on temperature. It can be evaluated with reasonable suppositions about the electronic wavefunctions, but depends on these and, of course, on the nature of the lattice. We are making numerical estimates of this second term and hope to publish the complete account elsewhere.

\section{Strachan}

A. M. Murray

Department of Natural Philosophy,

The University,

Aberdeen.

March 5.

${ }^{1}$ Kikuchi, S., and Nordheim, L., Z. Phys., 60, 652 (1930). ' Karplus, R., and Luttinger, J. M., Phys. Rev., 95, 1154 (1954).

\section{Birefringence Determination in Objects of Irregular Cross-sectional Shape and Constant Weight per Unit Length}

THE compensation method of obtaining the birefringence of an object seen in a polarizing microscope requires the evaluation of the optical path-difference produced by a known thickness. In elongated objects of irregular cross-sectional shape, for example, silk and viscose rayon filaments and fibres or filaments containing cavities, the thickness at a given point of compensation within the object cannot readily be determined ${ }^{1,2}$. The purpose of this communication is to describe a technique that has overcome this difficulty.

It is known that by means of a compensator suited to the optical character of the object and capable of producing an increase of path-difference across the field of view, a birefringent object can be contoured by fringes, the sharpness of which is similar to that obtaining in two-beam interference. It has been shown ${ }^{8,4}$ that by employing a modified photographic technique an appreciable sharpening of two-beam fringes can be obtained; Fig. 1 illustrates

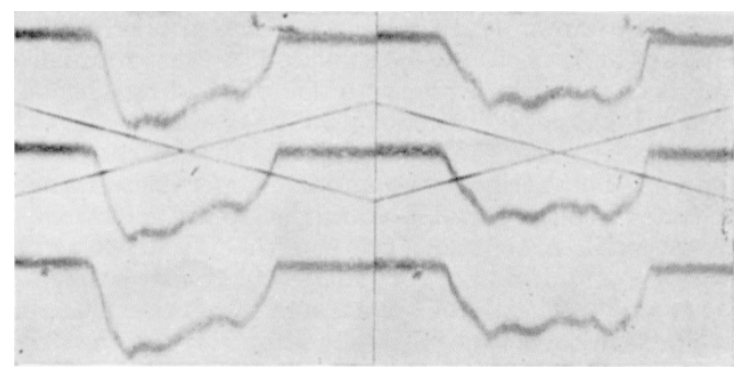

Fig. 1 